\title{
Educación superior y pueblos indígenas: marcos nacionales para contextualizar
}

\section{Inés Olivera Rodríguez}

Universidad Nacional Autónoma de México

inesolivera@gmail.com

\section{Gunther Dietz}

Universidad Veracruzana

guntherdietz@gmail.com

RESUMEN

Con la intensión de ofrecer el marco de referencia para comprender y comparar las experiencias canalizadas en este número, el siguiente texto presenta la situación de los jóvenes indígenas en la educación superior en México y Perú. Esta descripción contextual ha sido construida desde la comprensión de que todo lo avanzado en esta materia ha sido el resultado de procesos de configuración de una demanda, su traducción como política pública y su re-traducción en la gestión, aplicación o uso de la misma. De esta forma, el texto presenta los casos de México y Perú en dos ejes: la formulación de la demanda y la construcción de la oferta de educación superior intercultural o para pueblos indígenas.

Palabras clave: educación superior intercultural; educación superior y pueblos indigenas; universidades interculturales; politica de educación superior 


\section{Higher education and indigenous peoples: national contexts to place the experiences}

\section{$A B S T R A C T$}

In order to provide a contextual frame to understand and to compare the experiences analyzed in this issue, this introductory text presents the situation indigenous youth is facing in higher education in Mexico and Peru. This contextual presentation has been shaped by our conviction that what has been achieved is a result of a larger process of indigenous struggles and claims, their translation into public policy and its implementation inside higher education institutions. Accordingly, this text introduces the cases of Mexico and Peru through two dimensions: the emergence of the specific claims, on the one hand, and the respective construction of intercultural higher education for indigenous people, on the other hand.

Keywords: intercultural higher education, higher education and indigenous people, intercultural universities, higher education policy 
En el contexto latinoamericano, son sobre todo los movimientos indígenas y las organizaciones de profesionales procedentes de pueblos originarios quienes plantean — ya desde la década de 1970 — la necesidad de ofrecer una educación diversificada y apropiada para los contextos indígenas y afrodescendientes (Mato, 2014). Aunque la demanda de estos movimientos por educación superior tiene varias décadas, esta continúa padeciendo un doble rezago: por una parte, un evidente déficit de acceso (Schmelkes, 2011), y por otra, un rezago en cuanto a la pertinencia de la educación ofrecida. Las respuestas políticas han sido distintas en los diferentes países de América Latina, incluyendo tanto programas para la ampliación del acceso mediante becas y cuotas como la creación de sistemas educativos guiados por enfoques interculturales para la inclusión, no solo de los sujetos indígenas sino también de sus saberes. Así, en América Latina hemos visto surgir en las últimas décadas universidades e institutos indígenas, interculturales o comunitarios (Mato, 2008).

En esta sección hemos querido centralizar la reflexión en torno a este segundo caso: el de las experiencias de creación de propuestas interculturales de educación superior en dos países de la región: México y Perú. Los procesos son muy disímiles: México es el primero de la región en constituir todo un subsistema de universidades interculturales, mientras que el Perú es uno de los países con experiencia más reciente en este proceso, si se considera el estado de su implementación. Tanto en el Perú como en México, son los cambios constitucionales de la década de 1990 los que introducen la diversidad y multiculturalidad como característica nacional, tomando en cuenta, a su vez, el reconocimiento de derechos identitarios.

El arribo del neoliberalismo, que se caracteriza por el fin de la comprensión del Estado como garante de derechos para pasar a ser definido como administrador 
de servicios (Strathern, 2000), implicó la apelación a las políticas identitarias como estrategia para neutralizar las demandas sociales más radicales por derechos laborales, por tierra y por la manutención o ampliación de políticas de bienestar. Desde esta comprensión, las políticas identitarias aparecen como la traducción oficial de la demanda social, formulada de manera que no represente riesgos para los procesos económicos. En términos de las políticas de educación superior para pueblos indígenas, iniciadas con el arribo del nuevo milenio, podemos observar negociaciones entre la formulación de la demanda social, su traducción oficial en políticas públicas y la reinterpretación de estas en su puesta en práctica por actores indígenas y no indígenas.

\section{FORMULACIÓN E INSTITUCIONALIZACIÓN DE LA DEMANDA}

Tanto en México como en el Perú, aun tratándose de experiencias oficiales construidas desde el Estado, las políticas de educación superior intercultural son respuestas ante las demandas de los movimientos, lo que coloca la legitimidad de su existencia y de sus propuestas en constante debate. En las últimas tres décadas, los movimientos indígenas latinoamericanos han logrado pasar de una política reactiva en defensa de los territorios de los pueblos originarios - que reacciona a las nuevas olas de despojo producto del extractivismo - a una política proactiva de «judicialización» de sus demandas integrales de reconocimiento de sus territorios, de su cultura y de sus sistemas normativos propios (Postero y Zamosc, 2005). Como parte de esta nueva fase de las movilizaciones indígenas continentales, el derecho a la cultura y a la educación propias se ha reconocido oficialmente en los principales cánones jurídicos internacionales tanto como en las constituciones de los estados-naciones latinoamericanos. Ello se materializa, entre otros logros, en el establecimiento paulatino de nuevas instituciones universitarias o de nuevos incentivos de acceso de jóvenes indígenas a la educación superior, que ha sido y sigue siendo el nivel escolar de mayor exclusión de los pueblos originarios.

\section{Educación superior y jóvenes indígenas en México}

En México, la creación gubernamental de las universidades interculturales constituye una respuesta de las políticas - sobre todo federales - al desafío político que constituye en 1994 la aparición pública del Ejército Zapatista de Liberación Nacional (EZLN) en Chiapas y el posterior proceso de redefinición y renegociación de las relaciones entre el Estado-nación mexicano y los pueblos 
indígenas del país. En el transcurso de los diálogos — que culminaron en 1996 en la primera fase de los Acuerdos de San Andrés Larráinzar / Sacam Ch'en, aprobados por una comisión parlamentaria, pero nunca implementados por el gobierno federal—, una educación cultural y lingüísticamente pertinente para los jóvenes indígenas, accesible en sus comunidades y que abarcara el conjunto de los niveles educativos surgió como uno de los principales reclamos en materia lingüística, cultural y educativa (CDHMAPJ, 2000).

Por las implicaciones para la autonomía de los pueblos indígenas de Chiapas y de México, el gobierno federal del priista Ernesto Zedillo (1994-2000) logró bloquear la correspondiente iniciativa legislativa (Pérez Ruiz, 2001). Tras el histórico fin del régimen del «partido-Estado» en las elecciones presidenciales de 2000, el conservador Partido Acción Nacional (PAN), bajo la presidencia de Vicente Fox (2000-2006), retoma algunos de los reclamos plasmados en los Acuerdos de San Andrés, omitiendo cualquier reconocimiento a la autonomía territorial o política de los pueblos y comunidades indígenas, pero incluyendo en reformas constitucionales, en la Ley Indígena de 2001 y en leyes y reglamentos secundarios el reconocimiento del derecho de los pueblos indígenas de México a una educación intercultural y bilingüe que paulatinamente ha de abarcar todos los niveles escolares, desde la educación inicial hasta la educación superior.

Las nuevas instituciones gubernamentales encargadas de implementar esta política «postindigenista» o «neoindigenista» (Hernández, Paz y Sierra, 2004) en el ámbito educativo son la Coordinación General de Educación Intercultural y Bilingüe (CGEIB), creada en 2001 al interior de la Secretaría de Educación Pública (SEP) federal, y el Instituto Nacional de las Lenguas Indígenas (INALI), creado en 2003 como organismo gubernamental descentralizado. Junto con la Comisión Nacional para el Desarrollo de los Pueblos Indígenas (CDI), la dependencia federal heredera del Instituto Nacional Indígena, y con la Dirección General de Educación Indígena (DGEI) de la SEP, este nuevo entramado gubernamental es el responsable por el diseño e implementación de programas educativos concebidos como pertinentes para los pueblos indígenas. Aunque existen esfuerzos de descentralización, regionalización y consulta previa, libre e informada, la política educativa destinada a los pueblos indígenas de México sigue fuertemente centralizada en la SEP y enfocada sobre todo a los niveles de educación inicial y primaria, en los que se ha logrado establecer un subsistema de educación intercultural y bilingüe, mientras que en educación secundaria y postsecundaria únicamente hay intentos aislados de introducir alguna que otra asignatura regional en un currículum que 
sigue siendo homogéneo y homogeneizante para el conjunto del país y de los pueblos que lo componen (Dietz y Mateos Cortés, 2013).

Para aquellas y aquellos pocos jóvenes indígenas que logran concluir la educación media superior ${ }^{1}$, existen apenas oportunidades para acceder a la universidad. Son escasos los programas académicos adaptados a sus necesidades, a su origen rural o a su diversidad cultural y lingüística (Mateos Cortés, Mendoza Zuany y Dietz, 2013). Mientras que la gran mayoría de las universidades mexicanas, tanto públicas como privadas, se extienden únicamente a zonas urbanas y ofrecen carreras convencionales, algunas universidades públicas están generando determinadas medidas auxiliares de acompañamiento, apoyo o visibilización de estudiantes indígenas, con el objetivo de ampliar sus respectivas matrículas a estudiantes subrepresentados históricamente en las IES, como en el caso de los estudiantes indígenas. Se trata de programas de «acción afirmativa», en ese sentido anglosajón - affirmative action - , que originalmente son programas de apoyo, de cuotas de discriminación positiva, para visibilizar la presencia de estudiantes indígenas o afrodescendientes en las universidades convencionales. Uno de los programas de mayor envergadura en México es el programa «México Nación Multicultural» de la Universidad Nacional Autónoma de México (UNAM), creado en 2004 y en 2014 redefinido como Programa Universitario de Estudios de la Diversidad Cultural y la Interculturalidad (PUIC), que cuenta con cursos, talleres, tutorías y becas para estudiantes indígenas, además de un rico abanico de actividades de difusión del legado de diversidad cultural del país.

Existen programas parecidos, que pueden ser muy pequeños, como una oficina de atención a estudiantes indígenas, o algo más amplios, que operan en las principales universidades públicas del país. No obstante, lo que hasta la fecha no se ha logrado en las universidades convencionales es transitar de este reconocimiento de la diversidad de los estudiantes a una reforma del currículum, a una apertura hacia los saberes que aportan estos estudiantes provenientes de otras experiencias históricas, culturales o lingüísticas. Estos programas, por tanto, a menudo se limitan a acompañar a las y los estudiantes indígenas en su tránsito por una institución universitaria que sigue sin reconocer sus culturas, sus lenguas o sus saberes y que por ello les sigue siendo ajena. Al no afectar las estructuras hegemónicas monoculturales de estas universidades, los programas de acción afirmativa no han

La educación media superior en México corresponde a los últimos tres años de educación básica y obligatoria. Es el nivel que sigue a la educación secundaria y el requisito para el acceso a la educación superior. 
podido tener un impacto a largo plazo entre los saberes académicos y los saberes comunitarios. Se trata una vez más de iniciativas de «multiculturalismo neoliberal» en las que se maquillan relaciones de diversidad ahora visibilizadas, pero que limitan sustancialmente la plena participación del indio permitido (Hale, 2006).

Otra posibilidad que han ido obteniendo las y los jóvenes indígenas para acceder a la educación superior son aquellas — aún pocas - escuelas normales que se van diversificando para formar a docentes con un enfoque intercultural bilingüe, algo que proviene originalmente de la tradición del indigenismo y que hoy consiste en formar a futuros docentes de educación básica capaces de superar el añejo sesgo castellanizador del anterior bilingüismo de transición, donde se utiliza la lengua indígena en preescolar y en primaria, pero básicamente para transitar a un monolingüismo en castellano. La institución pionera en este ámbito ha sido la Universidad Pedagógica Nacional (UPN), que en las últimas décadas ha generado dos programas clave para la formación de docentes con enfoque intercultural y bilingüe: por un lado, ya desde 1982 la Unidad Ajusco de la UPN ofrece en la Ciudad de México una Licenciatura en Educación Indígena (LEI) para formar en un programa de tiempo completo a futuros docentes, directivos, supervisores, asesores pedagógicos y creadores de materiales educativos para el subsistema de educación básica indígena; por otro, las unidades descentralizadas de la UPN que operan en o cerca de las principales regiones indígenas del país ofrecen desde 1990 un programa educativo específico: la Licenciatura en Educación Preescolar y en Educación Primaria para el Medio Indígena (LEPEPMI), que cursan a tiempo parcial las y los docentes en servicio que ya obtuvieron su plaza en la educación básica, pero que aún no concluyeron sus estudios universitarios. A menudo, estas licenciaturas pedagógicas son la única oferta universitaria accesible a las y los jóvenes indígenas que desean continuar sus estudios sin abandonar sus comunidades de origen.

Es en este panorama difícil y desigual de acceso a la educación superior en el que desde 2001 se comienza a impulsar la creación de nuevas instituciones de educación superior: las universidades interculturales (Mateos Cortés y Dietz, 2013). Estas se han creado en distintas regiones indígenas de México, con la idea de ofrecer carreras con el enfoque de pertinencia cultural y lingüística para una determinada región. Aparte del acceso y de la ampliación de la cobertura educativa, uno de sus propósitos iniciales consiste en ofrecer a las y los jóvenes alternativas a la necesidad de emigrar de sus comunidades hacia la ciudad para continuar sus estudios; también se pretende generar nuevas oportunidades de empleo en las propias regiones. 


\section{Educación superior y jóvenes indígenas en Perú}

En el Perú, los espacios de construcción colectiva que involucran a las organizaciones para la definición de políticas son pocos y relativamente recientes. El momento más importante se encuentra en el gobierno de transición democrática de Paniagua (2000-2001) ${ }^{2}$, cuando se convoca una Comisión Especial Multisectorial para las Comunidades Nativas, integrada por los diversos ministerios y la Defensoría del Pueblo. Esta comisión estableció la formación de una Mesa de Diálogo y Cooperación, con participación de las organizaciones indígenas e instituciones interesadas, para la formulación de propuestas de solución para los problemas que afectan a los pueblos indígenas. Como explica Meentzen (2010), en este espacio participaron representantes del Estado y organizaciones indígenas ${ }^{3}$, además de consultores independientes y representantes de Asociaciones Civiles ${ }^{4}$ (Meentzen, 2010).

Para este trabajo fueron definidos ocho ejes de discusión, a partir de los cuales la comisión estableció objetivos y propuestas de compromisos del Estado, en el Plan de Acción para los asuntos Prioritarios entregado el 30 de junio de 2001 (Cueva Maza, 2007, p. 107). El segundo punto del Plan de Acción señala «ampliar la educación intercultural bilingüe a todas las comunidades nativas y en todos los niveles: inicial, primario, secundario y superior» (CEMCN, 2001, p. 27). En el Plan se explicitan como objetivos a corto plazo la necesidad de mejorar la formación de maestros EIB, la creación de plazas para profesores bilingües en todas las comunidades nativas, la contratación de los profesores bilingües indígenas ya formados y la ampliación de la educación bilingüe y el enfoque intercultural a todos los niveles del sistema educativo en el país. El objetivo 5 del eje de educación EIB en el mismo documento demanda la participación de las

2 Se denomina gobierno de transición al gobierno de Valentín Paniagua entre noviembre del año 2000 y julio de 2001, gobierno establecido ante la fuga y posterior renuncia por fax de Alberto Fujimori, hoy con seis sentencias de cárcel por los crímenes: usurpación de funciones, peculado, asesinato, secuestro, corrupción y desviación de fondos.

3 La Asociación Interétnica para el Desarrollo de la Selva Peruana (Aidesep), la Asociación Regional de los Pueblos Indígenas de la Selva Central (ARPI), la Federación Nativa de Madre de Dios (Fenamad), la Confederación de Nacionalidades Amazónicas del Perú (Conap) y la Coordinadora Agroforestal Indígena y Campesina del Perú (Coicap).

4 Conservación Internacional (CI), la Asociación para la Conservación del Patrimonio de Cutivireni (ACPC), el Centro para el Desarrollo del Indígena Amazónico (Cedia), Apeco, el Centro Amazónico de Antropología y Aplicación Práctica (CAAAP) y el Instituto de Bien Común (IBC). 
organizaciones indígenas para el diseño y aplicación de las políticas vinculadas con la EIB en todos los niveles educativos (CEMCN, 2001).

El mismo año, el gobierno de transición aprobó la constitución de la Mesa de Diálogo Permanente para la solución de los problemas de las Comunidades Indígenas de la Amazonía Peruana (Cueva Maza, 2007). Según su decreto de creación, esta mesa tenía como objetivos evaluar la aplicación del Plan de Acción, establecer Mesas de Diálogos Regionales y elaborar, y proponer y coordinar la aplicación de un Plan Integral para el Desarrollo de las Comunidades Nativas. El gobierno entrante, de Alejandro Toledo (2001-2006), crea en 2001 la Comisión Nacional de los Pueblos Andinos, Amazónicos y Afroperuanos (Conapa) sin vinculación con las recomendaciones del Plan de Acción, ni con la Mesa de Diálogo. De esta forma, la Mesa de Diálogo permanente no se constituyó y las recomendaciones y compromisos del Plan de Acción quedaron olvidados hasta el año 2009 cuando, como resultado de los levantamientos amazónicos contra la firma del TLC y las reformas legales que preparaban su aplicación, que tuvieron su punto más álgido en la represión violenta del levantamiento en Bagua el 10 de junio de 2009, se crea el Grupo Nacional de Coordinación para el Desarrollo de los Pueblos Amazónicos (Agurto, 2011).

Como señala Meentzen (2010), la Mesa de Diálogo de 2001 se caracterizó por una convocatoria democrática que pretendió — y logró en buena medida — la inclusión de las organizaciones indígenas - por ejemplo, al no exigir credenciales de representación, ni evaluar la aceptación de participación, sino, por el contrario, manteniéndose como espacio abierto para la participación ciudadana-, pero la Mesa de Diálogo convocada en el gobierno de García en 2009 no tuvo este objetivo ni actuó de esta forma, siendo que la participación fue ampliamente restringida, contando con muy poca representación de las organizaciones indígenas y sociales ${ }^{6}$.

A pesar de la poca representatividad de este proceso, la mesa elaboró la Propuesta Nacional para el Desarrollo Amazónico, que retoma lo establecido en el Plan de 2001 en lo concerniente a la ampliación de la educación intercultural bilingüe a todas las comunidades nativas y en todos los niveles: inicial, primaria, secundaria y superior, la mejora de la formación y capacitación de los maestros bilingües y otros profesionales indígenas y la necesidad de generar avances con

Inicialmente la Conapa no incluye a los pueblos afrodescendientes; recién en 2003 se hace una reforma del nombre para su inclusión.

6 Cabe señalar que no solo la Mesa de Diálogo, sino todo el segundo gobierno de García (2006-2011), se caracterizó por la observancia, tanto de instancias nacionales - Defensoría del Pueblo- como internacionales, la OIT. 
relación a la oferta de educación superior para jóvenes indígenas con tres medidas: reestructurar la Universidad Intercultural de la Amazonía (UNIA) fundada en el año 2000, crear programas especiales en cada universidad nacional para atender a los jóvenes indígenas universitarios y establecer un programa de becas para jóvenes indígenas de toda la Amazonía. Nuevamente está presente, de forma transversal, la demanda por participación de las organizaciones indígenas a través de consulta para la formulación de políticas pertinentes (Mesa de Trabajo No 4, 2009, pp. 12-20).

Estas demandas iniciaron su implementación a partir del año 2010, con cambios institucionales importantes en el gobierno, entre los cuales destacan la creación del Ministerio de Cultura en 2010, que acoge a su interior al Viceministerio de Interculturalidad, donde funcionan la Dirección General de Ciudadanía Intercultural; la Dirección de Políticas para Pueblos Indígenas y el Grupo de Trabajo de Pueblos Indígenas (GTPI); la Nueva Ley Universitaria de 2014, que establece el control desde el Ministerio de Educación de las universidades, lo que dio un importante empuje para la implementación de las universidades interculturales, creadas en papel en 2011, pero sin funcionamiento, y el Programa Nacional de Becas (Pronabec), que desde 2012 comienza a incluir modalidades de becas para jóvenes indígenas. De esta forma, es posible rastrear los avances actuales en política para acceso, permanencia y pertinencia de y a la educación superior por jóvenes indígenas, hasta las propuestas de las organizaciones vertidas en los escasos espacios en los cuales fueron convocadas.

\section{LA CONSTRUCCIÓN DE OFERTAS DE EDUCACIÓN SUPERIOR INTERCULTURAL}

Ante la demanda, parte de la respuesta ha sido similar en ambos países: la creación de universidades interculturales, aunque sus principios y objetivos no sean necesariamente los mismos y sean también tan disimiles sus avances. Estas instituciones aparecen como una construcción que parte del reconocimiento de la necesidad de establecer una oferta educativa distinta, transformando los espacios formativos de educación superior, más allá de garantizar el acceso de jóvenes indígenas a modelos tradicionales de formación profesional. En este sentido, comienzan a perfilarse como los espacios de mayor acogida de jóvenes indígenas en la educación superior, además de representar espacios de construcción crítica para la sociedad en general. Sin embargo, existen también otro tipo de ofertas educativas, anteriores y aún vigentes de acceso a la educación superior de jóvenes indígenas, como la formación pedagógica. 
Tanto en el Perú como en México, la nueva educación superior intercultural surge como un intento gubernamental de ampliar la oferta educativa a nivel universitario, por un lado, y de repensar y reformular los saberes académicos a partir de su encuentro o «encontronazo» con los saberes indígenas comunitarios, por el otro. Las carreras ofertadas, los modelos educativos desarrollados y el papel asignado a los conocimientos indígenas en cada una de estas instituciones interculturales son por ello el resultado de las evoluciones y tensiones entre las demandas indígenas, las imposiciones gubernamentales y las lógicas académicas, estas dos últimas fuertemente influenciadas por imposiciones del capitalismo neoliberal.

\section{Las universidades interculturales mexicanas}

Después de las elecciones presidenciales del año 2000, que ponen fin a la secular tradición de partido-Estado, el nuevo gobierno federal, de orientación conservadora, reconoce oficialmente la «educación intercultural y bilingüe» como una nueva prioridad política, aun cuando no accede a reconocer el derecho a la autonomía indígena ${ }^{7}$. El reconocimiento oficial de la «educación intercultural y bilingüe» por el gobierno de Fox como una nueva prioridad política ha implicado por un lado una consolidación de los arriba mencionados programas de formación docente indígena con enfoque intercultural y bilingüe, que ofrecen la UPN y varias escuelas normales. Por otro lado, generó la creación a partir de 2003 de las primeras universidades interculturales públicas. Desde entonces, estas nuevas instituciones de educación superior, que articulan su propia red universitaria ${ }^{8}$, surgen a partir de convenios entre la arriba mencionada CGEIB federal y los respectivos gobiernos de cada uno de los estados en los que se ubica la respectiva institución de nueva creación: en la región mazahua del centro de México (Universidad Intercultural del Estado de México, fundada en 2004; cfr. http://www.edomexico.gob.mx/uiem/index.htm), en la región chol de Tabasco (Universidad Intercultural del Estado de Tabasco, fundada en 2005; cfr. http:// www.uiet.edu.mx/), en la ciudad multiétnica de San Cristóbal de la Casas, Chiapas (Universidad Intercultural de Chiapas, desde 2005; cfr. http://www.unich.edu. $\mathrm{mx} /$ ), en la región nahua y totonaca de la sierra norte de Puebla (Universidad Intercultural del Estado de Puebla, desde 2006; cfr. http://www.uiep.edu.mx/),

7 Resumimos aquí un panorama de universidades interculturales desarrollado con más detalle en Dietz (2017).

8 REDUI (cfr. http://www.redui.org.mx/), hoy denominada Asociación Nacional de Universidades Interculturales (ANUI). 
en la región maya de la península de Yucatán (Universidad Intercultural Maya de Quintana Roo, desde 2007; cfr. http://www.uimqroo.edu.mx), en la montaña nahua de Guerrero (Universidad Intercultural de Guerrero, fundada desde 2007; cfr. http://www.uieg.edu.mx), en la región purépecha de Michoacán(Universidad Intercultural Indígena de Michoacán, fundada desde 2007; cfr. http://www.uiim. edu.mx) y en la región hñähñu (otomí) de Hidalgo (Universidad Intercultural del Estado de Hidalgo, fundada en 2012; cfr. http://es-la.facebook.com/public/ Universidad-Intercultural-Del-Estado-De-Hidalgo-Uiceh).

Además de estas universidades de nueva creación, hay instituciones preexistentes que se han unido al programa federal. En primer lugar, la decana de las iniciativas de educación superior indígena en el país, la Universidad Autónoma Indígena de México, hoy denominada Universidad Autónoma Intercultural de Sinaloa, localizada en la región yaqui y mayo de Sinaloa, se remonta al año 1982, pero en 2005 se une a la red patrocinada por la CGEIB (cfr. http://uais.edu.mx/). En segundo lugar, la Universidad Veracruzana, una universidad pública preexistente, inicia su propio programa, el de la Universidad Veracruzana Intercultural, que desde su fundación en 2005 opera en las regiones veracruzanas de la Huasteca, el Totonacapan, la Sierra de Zongolica y las Selvas (cfr. http://www.uv.mx/uvi). Finalmente, el sistema privado de universidades pertenecientes a la Orden Jesuita funda en su propia universidad cultural, creando en la región ayuuk (mixe) de Oaxaca el Instituto Superior Intercultural Ayuuk (cfr. http://www.isia.edu.mx/).

A pesar de sus diferencias en cuanto a origen y composición lingüística y cultural, algunas características compartidas van surgiendo como definitorias de estas nuevas instituciones: no están dirigidas solo a los pueblos indígenas, sino que atienden preferencialmente a regiones indígenas y a poblaciones escolares excluidas históricamente. Por consiguiente, no es la etnicidad indígena, sino la interculturalidad y la diversidad lo que conforma el fundamento de sus actividades educativas. A diferencia de programas explícitamente diseñados como de acción afirmativa, tales como la iniciativa Pathways to Higher Education promovida desde 2001 por la Fundación Ford, las universidades interculturales no recurren a «lo indígena» como un criterio de acceso (Schmelkes, 2011). En cuanto a la oferta educativa, las universidades interculturales enfrentan el reto de tener que diseñar programas académicos completamente nuevos y adaptados al entorno local tanto en términos lingüísticos y culturales como en relación con la situación económica y de empleabilidad regional. Para ello, se procuró evitar el sesgo indigenista que limitaba la promoción de jóvenes indígenas a profesiones pedagógicas o agronómicas, ofertas educativas que — como las arriba mencionadas 
Licenciatura en Educación Indígena y Licenciatura en Educación Preescolar y en Educación Primaria para el Medio Indígena - siguen predominando en las regiones indígenas para generar intermediarios verticalmente útiles al Estado.

Por otro lado, los programas de estudios demasiado convencionales, centrados en empleos únicamente urbanos, industriales o de servicios, únicamente hubieran incrementado la presión migratoria para que los jóvenes se trasladaran a las ciudades. Considerando estas prioridades, y en estrecha relación con la CGEIB, las universidades interculturales han ido desarrollando y experimentando con una gama cada vez más amplia de programas de licenciaturas, que incluyen carreras como lengua y cultura, comunicación intercultural, gestión intercultural para el desarrollo, desarrollo sostenible, turismo, patrimonio, gestión municipal, así como últimamente salud intercultural y derecho intercultural.

A diferencia de las carreras convencionales, aquellas que se implementan en las universidades interculturales se caracterizan por procurar mantener desde un inicio una estrecha relación entre la enseñanza-aprendizaje áulica, por un lado, y la vinculación comunitaria, por otro. Varios proyectos de desarrollo han sido iniciados y piloteados por profesores y estudiantes desde dentro de estas universidades, que comienzan a fungir como «incubadoras» de proyectos que posteriormente son aplicados por y transferidos a actores locales, tales como organizaciones no gubernamentales, autoridades municipales y otras contrapartes. A través de estas actividades, los estudiantes adquieren conocimientos prácticos y estratégicos, que pretenden formarlos como un nuevo tipo de mediador o traductor «desde abajo y desde dentro» y no — como en la época del indigenismo - «desde arriba y desde fuera». Las inestables características de los mercados laborales de las regiones rurales e indígenas obligan a las y los estudiantes a desarrollar redes diversificadas y altamente flexibles de contrapartes, a través de las cuales a veces logran encontrar un empleo y a veces se autoemplean o logran fundar sus propias empresas, cooperativas u ONG.

\section{La educación superior intercultural en el Perú}

En el Perú existen, con decreto de fundación, cuatro universidades interculturales: la Universidad Nacional Intercultural de la Amazonía (UNIA), ubicada en la región Ucayali y creada por ley 27250 del 10 de diciembre de 1999; la Universidad Nacional Intercultural de la Selva Central Juan Santos Atahualpa (UNIJSA), ubicada en la región Junín y creada por ley 29616 el 17 de noviembre de 2010; la Universidad Nacional Intercultural de Quillabamba (UNIQ), ubicada en la región 
Cusco y creada por ley 29620 el 17 de noviembre de 2010, y la Universidad Nacional Intercultural Fabiola Salazar Leguía (Unibagua), ubicada en la región Amazonas y creada por ley 29614, el 12 de noviembre de 2010. Sin embargo, solo una de ellas funciona y recibe estudiantes desde el año 2005: se trata de la UNIA, con autorización de funcionamiento emitida por la Conafu en el año $2003^{9}$.

Como se mostró en acápites anteriores, la demanda de las organizaciones indígenas por educación superior resaltaba no solo las garantías para el acceso sino también la pertinencia de la educación ofrecida (Itacab, 2017). En las discusiones articuladas en torno a las universidades interculturales, la pertinencia cultural se entiende como la posibilidad de educarse en la universidad desde los saberes y formas de aprender de las comunidades indígenas, lo que incluye la presencia tanto de las lenguas como de sabios indígenas y comunitarios en los procesos de aprendizaje. Es desde esta demanda que se definió la creación de un sistema nuevo universitario como proceso paralelo a políticas de acción afirmativa en universidades convencionales; sin embargo, y como se verá, no es desde esta comprensión que se articuló su construcción en sus primeros años de existencia.

La UNIA se crea en el proceso de campaña electoral para la segunda reelección de Fujimori, como concesión en la búsqueda por apoyo social, sin la intención de planificar su funcionamiento. Ante la fuga y renuncia de Fujimori, su implementación queda paralizada y solo será retomada por el gobierno de Toledo (2001-2006) en respuesta a la demanda vertida por el Plan de Acción de 2001. Distintos intereses y agendas políticas generaron la creación de las otras tres universidades interculturales, lo que hay que considerar es que la existencia de la Propuesta Nacional para el Desarrollo de la Amazonía (2009), que implicaba un compromiso nacional para la transversalización y mejora de la educación intercultural en todos los niveles, proveyó un marco desde el cual fue posible cumplir con ciertos compromisos políticos de crear nuevas universidades públicas. Por otro lado, los eventos acontecidos en Bagua ese mismo año, y la gran caída de popularidad del gobierno de García, en gran medida por dichos eventos, generó un escenario provechoso para políticas de interculturalidad, principalmente con pueblos amazónicos.

$\mathrm{Al}$ analizar los retos y problemas con los que se enfrentan estas universidades para cumplir su objetivo e iniciar funcionamiento, un tema central es la definición

9 En el marco de la ley universitaria anterior. Actualmente continúa funcionando, pero se encuentra en proceso de licenciamiento ante la Superintendencia Nacional de Educación Superior Universitaria (Sunedu), como exigido por la Ley Universitaria de 2014. 
y transversalización del enfoque intercultural (Itacab, 2017; DGESU, 2017). De esta forma, se señala que la oferta educativa definida en los decretos de creación no considera el enfoque intercultural, ni por la elección de las carreras, ni por el diseño de su malla curricular y contenidos. Esto se explica porque bajo ley universitaria anterior (ley 23733), en el marco de la cual fueron creadas estas instituciones, el Consejo Nacional para la Autorización y Funcionamiento de Universidades (Conafu), reemplazado por Sunedu, aprobó los planes de desarrollo institucional (PDI) sin la consideración de ningún criterio de enfoque intercultural (Itacab, 2017). Esta ausencia del enfoque intercultural es evidente tanto en los planes de estudio y las mallas curriculares como en la definición de los perfiles docentes; así, la UNIA declara que de sus 63 docentes de tiempo completo ninguno es hablante de lengua indígena, ni tiene formación en interculturalidad (Castañeda Chávez, 2017).

La universidad Juan Santos Atahualpa orienta su crítica también a la dificultad de incluir a los sabios indígenas en los procesos de la universidad, lo que en parte atribuye a las normas de licenciamiento establecidas por la Sunedu. Como estrategia de respuesta se ha intentado incluir a los sabios indígenas y representantes de organizaciones en la docencia, no áulica, de la universidad; la segunda estrategia ha sido disponer que los profesores que han ganado plaza «tengan que someterse a un taller de tres meses sobre interculturalidad, conocimiento intercultural e investigación intercultural» (Pomachagua Paucar, 2017, p. 227).

De esta forma, los avances en el Perú en términos de una oferta pública de educación superior intercultural son aún declarativos e incipientes. En la práctica no se ha construido un modelo educativo para la universidad intercultural que garantice la inclusión de los saberes, lenguas y procesos cognitivos de los jóvenes indígenas; tampoco se ha logrado garantizar la participación comunitaria y de organizaciones indígenas de forma que sea posible la consulta permanente para el diseño y aplicación del dicho modelo. En este escenario, aunque las transformaciones en las políticas y la organización estatal han sido importantes en los últimos diecisiete años, en la práctica el principal avance en este campo continúa estando del lado de la acción de organizaciones sociales que implementan la oferta de educación superior intercultural.

Por detrás de esta oferta intercultural, es y ha sido central el trabajo de Aidesep. En el Perú, la formación docente en EIB se inicia de forma experimental en 1993 con el Modelo Curricular de Formación Docente en Educación Intercultural Bilingüe (Mofeib) y comienza a ofrecerse recién en el año 2000 en cinco institutos superiores pedagógicos andinos (Córdova Cusihuamán, 2012). 
Sin embargo, desde las organizaciones, Aidesep había comenzado este trabajo al impulsar y acompañar desde 1988 la creación del Programa de Formación de Maestros Bilingües de la Amazonía Peruana (Formabiap) (Trapnell, 2008). Esta experiencia continúa vigente y se ha expandido al influir en otros centros de formación EIB.

El segundo caso importante de oferta educativa superior técnica con enfoque intercultural es la recientemente aprobada carrera en salud intercultural, detrás de cuya implementación también estuvo Aidesep. En el año 2012, Aidesep, el instituto Joaquín Reátegui y la Dirección Regional de Salud de Loreto presentaron ante el Minedu el expediente 0212184-2012, solicitando la creación de la nueva carrera de «Enfermería Técnica Intercultural Bilingüe Amazónica». Esta carrera obtuvo autorización de oferta y funcionamiento a través del oficio 124, del 1 de abril del año 2015 (Chávez, Yon y Cárdenas, 2015, pp. 20-21), luego de lo cual ha tenido ingreso en 2016 y 2017 en el IESTP José Reátegui de Nauta con alumnos Achuar (Cárdenas y Pesantes, 2017).

\section{BALANCE}

Este número busca ilustrar la situación de la educación superior con relación a jóvenes indígenas e interculturalidad en Perú y México. Considerando que este tema será trabajado como uno de los siete ejes en la Conferencia Regional de Educación Superior de la Unesco (CRES), a realizarse en junio de 2018, el presente resulta un tiempo demandante de miradas y enfoques comparativos entre las experiencias regionales que nos permitan avanzar en la construcción de distintas ofertas de educación superior intercultural encaminadas hacia la transformación de la educación superior con criterios de pertinencia cultural y lingüística, que coadyuven a saldar deudas con aquellas comunidades que históricamente han sido excluidas, discriminadas y estigmatizadas por las universidades latinoamericanas.

En la sección temática que presentamos en este número se incluyen cuatro trabajos que analizan la experiencia peruana. La contribución de Oscar Espinosa presenta un panorama analítico en torno a las distintas propuestas de educación superior y jóvenes indígenas, considerando algunas políticas de acción afirmativa en universidades convencionales, programas de formación técnica diseñados en el enfoque intercultural y las universidades interculturales. Analizando la construcción de la carrera técnica en salud intercultural -impulsada por Aidesep-y los impactos logrados en veintiocho años de experiencia del programa de formación de maestros bilingües (Formabiap), también impulsado por Aidesep, 
se incorporan a este número las contribuciones de Cynthia Cárdenas, María Amalia Pesantes y Alfredo Rodríguez, y la de Lucy Trapnell. Finalmente, sobre el acceso de jóvenes indígenas a las universidades convencionales, esta sección presenta el aporte de Alejandra Hidalgo Bonicelli que analiza las dificultades de jóvenes indígenas estudiantes de una universidad pública y convencional en la Amazonía peruana.

Las contribuciones sobre estudiantes y egresados/as de universidades interculturales mexicanas que aportan a este dossier Inés Olivera Rodríguez para el caso de la Universidad Veracruzana Intercultural, Sergio Enrique Hernández Loeza para el caso de la Universidad Intercultural del Estado de Puebla y Alejandro Mira Tapia para el caso del Instituto Intercultural Ñöñho conforman noveles estudios empíricos que ilustran y detallan este ambiguo panorama de empleabilidad precaria, empoderamiento limitado y migraciones cualificadas de nuevos actores letrados surgidas de la educación superior intercultural mexicana. Estas experiencias resultan similares a las que retrata y analiza Yasmani Santana Colín para quienes egresan de la licenciatura en Educación Indígena de la UPN. Por su parte, la contribución de Francisco Rosado May sobre el caso de la Universidad Intercultural Maya de Quintana Roo se enfoca en los impactos sociales y económicos que estas nuevas instituciones de educación superior comienzan a causar en sus entornos locales y regionales.

\section{REFERENCIAS BIBLIOGRÁFICAS}

Agurto, J. (2011). Perú: Desafíos de la agenda institucional para pueblos indígenas del nuevo gobierno. Servindi. Recuperado de https://www.servindi.org/actuali$\mathrm{dad} / 50161$

Cárdenas, C. y M.A. Pesantes (2017). Entrecruzando ríos: sistematización de la propuesta pedagógica de formación de enfermeros técnicos en salud intercultural de AIDESEP. Lima: IEP-AIDESEP.

Castañeda Chávez, V. (2017). La Universidad Nacional Intercultural de la Amazonía Peruana (UNIA). En Itacab (ed.), Educación superior intercultural indígena en el Perú y América Latina: aproximaciones, experiencias y desafíos (pp. 217222). Lima: Itacab.

CDHMAPJ [Centro de Derechos Humanos Miguel Agustín] (2000). Derecho de los pueblos indigenas: experiencias, documentos y metodologías. México: $\mathrm{CDH}-$ MAPJ. 
Chávez, C., C. Yon y C. Cárdenas (2015). El aporte de los egresados del Programa de Formación de Enfermeros Técnicos en Salud Intercultural Amazónica de AIDESEP a la salud intercultural. Estudio de caso en dos comunidades de Amazonas. Documento de trabajo $\mathrm{N}^{\mathrm{O}} 222$. Lima: IEP.

Córdova Cusihuamán, G. (2012). Educación superior en el Perú: los pueblos indígenas y afroperuanos y las políticas públicas e institucionales. En D. Mato (coord.), Educación superior y pueblos indígenas y afrodescendientes en América Latina. Normas, políticas y prácticas (pp. 325-359). Caracas: UNESCO-IESALC.

Cueva Maza, N. (2007). La salud de los indígenas en aislamiento En IWGIA (ed.), Pueblos indígenas en aislamiento voluntario y contacto inicial en la Amazonía y el Gran Chaco. Copenhague: Tarea - IWGIA.

Dietz, G. (2017). Introducción: las universidades interculturales en México, sus retos y perspectivas. En González, F., F. Rosado-May y G. Dietz (eds.), La gestión de la educación superior intercultural: retos y perspectivas de las universidades interculturales (pp. 21-31). Chilpancingo, Gro.: Trinchera, Universidad Autónoma de Guerrero y El Colegio de Guerrero, A.C.

Dietz, G. y L.S. Mateos Cortés (2013). Interculturalidad y educación intercultural en México: un análisis de los discursos nacionales e internacionales en su impacto en los modelos educativos mexicanos. México: SEP-CGEIB.

Grupo Nacional de Coordinación para el Desarrollo de los Pueblos Amazónicos (2009). Mesa $N^{\mathrm{o}}$ 4: Propuesta Nacional de Desarrollo Amazónico (Acta del 22 de junio de 2009). Lima.

Hale, Ch.R. (2006). Más que un indio: More than an Indian: Racial ambivalence and neoliberal Multiculturalism in Guatemala. Santa Fe: School of American Research Press.

Hernández, R. A., S. Paz y M.T. Sierra (2004). Introducción. En R.A. Hernández, S. Paz y M.T. Sierra (eds.), El Estado y los indigenas en tiempos del PAN: neoindigenismo, legalidad e identidad (pp. 7-25). México: CIESAS y Miguel Ángel Porrúa. https://doi.org/10.4000/books.ifea.4963

Itacab (2017). Educación superior intercultural indígena en el Perú y América Latina: aproximaciones, experiencias y desafios. Lima: ITACAB.

Mateos Cortés, L.S. y G. Dietz (2013). Universidades Interculturales en México. En M. Bertely Busquets, G. Dietz y G. Díaz Tepepa (coords.), Multiculturalismo y educación 2002-2011 (pp. 349-381). México: COMIE y ANUIES.

Mateos Cortés, L.S., G. Mendoza Zuany y G. Dietz (2013). Diversidad e interculturalidad en la educación superior convencional. En M. Bertely Busquets, G. Dietz y G. Díaz Tepepa (coords.), Multiculturalismo y educación 2002-2011. México: COMIE y ANUIES. 
Mato, D. (2008). Diversidad cultural e interculturalidad en educación superior. Problemas, retos, oportunidades y experiencias en América Latina. En D. Mato (coord.), Diversidad cultural e interculturalidad en educación superior. Experiencias en América Latina (pp. 21-82). Caracas: IESALC-Unesco.

Mato, D. (2014). Universidad indígena en América Latina. Experiencias, logros, problemas, conflictos y desafíos. ISEES, 14, julio-diciembre, 17-45.

Meentzen, A. (2010). Experiencias de diálogo y la aplicación del Convenio 169 de la OIT en el Perú (Estudios de caso). Recuperado de http://infoindigena.servindi. org/images/Publicaciones_generales/Peru-publicaciones/Experiencias-dilogo-yla-aplicacin-del-Convenio-169-de-la-OIT-en-Per.pdf

Pérez Ruiz, M.L. (2001). Los acuerdos de San Andrés en su contexto político y militar. Boletín CEAS, 5, 11-16

Pomachagua Paucar, J. (2017). Universidad Nacional Intercultural de la Selva Central Juan Santos Atahualpa. En Itacab (ed.), Educación superior intercultural indigena en el Perú y América Latina: aproximaciones, experiencias y desafios (pp. 223-226). Lima: Itacab.

Postero, N. y L. Zamosc (eds.) (2005). La lucha por los derechos indígenas en América Latina. Quito: Abya-Yala.

Schmelkes, S. (2011). Programas de formación académica para estudiantes indígenas en México. En S. Didou Aupetit y E. Remedi Allione (coords.), Educación superior de carácter étnico en México: pendientes para la reflexión (pp. 65-78). México, D.F: Senado de la República, CINVESTAV.

Strathern, M. (2000). Introducción: nuevas rendiciones de cuentas. Estudios antropológicos en auditoria, ética y la academia. En M. Strathern (coord.), Audit cultures. Anthropological studies in accountability, ethics and the academy (pp. 1-18). Londres: Routledge. https://doi.org/10.4324/9780203449721

Trapnell, L. (2008). La experiencia del Programa de Formación de Maestros Bilingües de la Amazonía Peruana. En D. Mato (coord.), Diversidad cultural e interculturalidad en educación superior. Experiencias en América Latina, (pp. 403-412). Caracas: Unesco-IESALC. 